\title{
INFLUENCE OF MIX PROPERTIES ON THE PERMANENT DEFORMATION CHARACTERISTICS OF BITUMINOUS MIXTURES
}

\author{
B.Bala Subrahmanyam ${ }^{1}$, P.Gopi ${ }^{2}$, M.Balraj Naik ${ }^{3}$ \\ ${ }^{l}$ PG Student, Department of Civil Engineering, M.V.R College of Engineering and Technology, Andhra Pradesh, \\ India \\ ${ }^{2}$ Asst. Professor, Department of Civil Engineering, M.V.R College of Engineering and Technology, Andhra Pradesh, \\ India \\ ${ }^{3}$ Asst. Professor, Department of Civil Engineering, M.V.R College of Engineering and Technology, Andhra Pradesh, \\ India
}

\begin{abstract}
India is one of the countries having the largest road network where majority of the roads are paved with bitumen-based macadamized roads with a thin bituminous surfacing or a premix carpet as a wearing course. Bituminous roads are mainly composed of naturally available aggregates and hot bitumen/asphalt. In these bituminous pavements Permanent deformation is one of the main failure modes in pavement structures subjected to mechanical loading. Load acting by the vehicle wheel on the pavement of a road result in permanent deformation. Permanent deformations, primarily in the form of ruts in the form of corrugations, tracks, imprints, and shoving are one of the basic asphalt pavement damages impairing its service properties. Ruts may cause vehicles to skid during rains in that case they are more dangerous than the listed above. So as to increase the pavement performance there is a need to increase the quality of pavement in the present study an attempt has been made to evaluate the permanent deformation characteristics of a bitumen mixture with different mix proportions. Wheel tracking testing equipment used to evaluate the rutting performance of bituminous mixtures. By the data of wheel tracking testing can identify the pavement performance and to improve the quality of a road. By developing a model can understand the performance of VG30 bitumen under different temperature conditions.
\end{abstract}

Keywords: Permanent Deformation, Bituminous Mix Influence, Rutting Characteristics, Rutting Model By Regression Analysis Etc...

\section{INTRODUCTION}

Bituminous mixes have been exposed to greater stresses because of the increasing traffic volumes, higher axle loads than the allowable loads and high type pressures. These increasing stresses pose different forms of failures like fatigue, permanent deformation and surface wear in the early years of the pavement service life. One of the most important forms of distress is the permanent deformation along the wheel paths of bituminous layers which may influence the steering of vehicles at high speeds and hydroplaning.

The basic constituents of bituminous mixes are binder, air voids and aggregates. These three constituents separately or together can affect the rutting in bituminous layers. Aggregate occupy 90 percentages of the volume in bituminous mixes and their shape and texture will influence the aggregate interlocking. Stiffness of the binder plays a major role in rutting and low viscosity binder produces less stiff binder. Air voids in mix can be controlled by compaction during construction of pavements. The mixes having lesser air voids undergo shear failure.

As truck speeds are decreased on a Hot Mix Asphalt pavement, the increase of stresses is because of longer contact times with the pavement. The increase in stresses increases the rutting probability. Higher tyre pressures create higher stresses in the pavement.

\subsection{Rutting or Permanent Deformation}

Permanent deformation is one of the most important distresses in asphalt pavements in that permanent deformation Rutting is one of the most important form. In now a day the truck loading condition is increases Deformation in the form of rutting in flexible pavement has become the dominant mode of failure. In general rutting has been classified in to three causes in asphalt pavements: subgrade permanent deformation, accumulation of permanent 
deformation in the asphalt surfacing layer, and wear of pavements caused by studded tires. Rutting can caused by all of three acts listed above in combination, mean the summation of deformation in all layers of pavement and wear from studded tires.

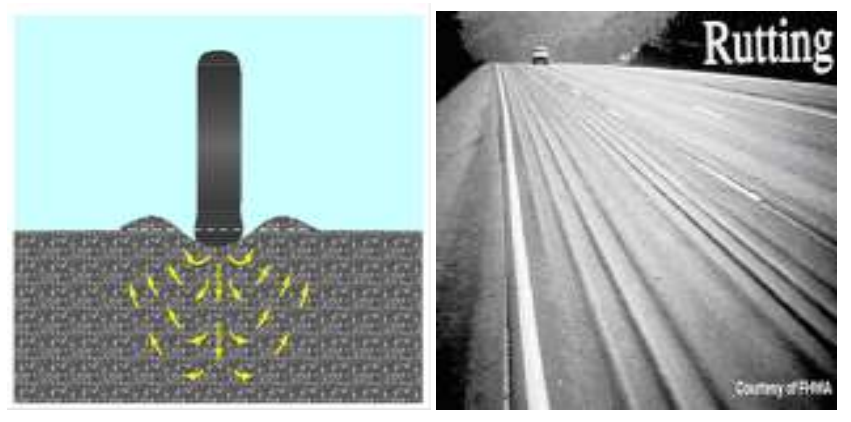

\section{METHODOLOGY}

This study concerned to know the changes in rut depth in asphalt pavement by varying test conditions like temperature, air voids, binder types, type of binder grade and binder content. In methodology a detailed review regarding the influence of mix proportions on permanent deformation in asphalt mixtures are discussed.

The methodology divided into two stages: experimental work and analytical work. Mixes like bituminous concrete (BC), dense bituminous concrete (DBM) were prepared by varying the type of binder, binder content, air voids gradation of BC and DBM is used in this study. Mixes were prepared using ASTM D 6926-10: The slabs are prepared by compacting the mixes using roller compactor. The asphalt mixes were tested for rutting at various temperatures (40, 50,60 , and $70^{\circ} \mathrm{C}$ ) and at standard load. The rut depth under these conditions is determined using Wheel Tracking Test (WTT) which was developed by British National Rail Road Institute (BNRRI). The performance of mixes is observed by varying test temperatures, type of binder, and binder content.

This study can help to find the correlation of mix properties with permanent deformation in asphalt mixtures. After correlation of the properties, a new model is developed. The performance of asphalt mixes is to evaluated by predicting the rut depth using obtained model.

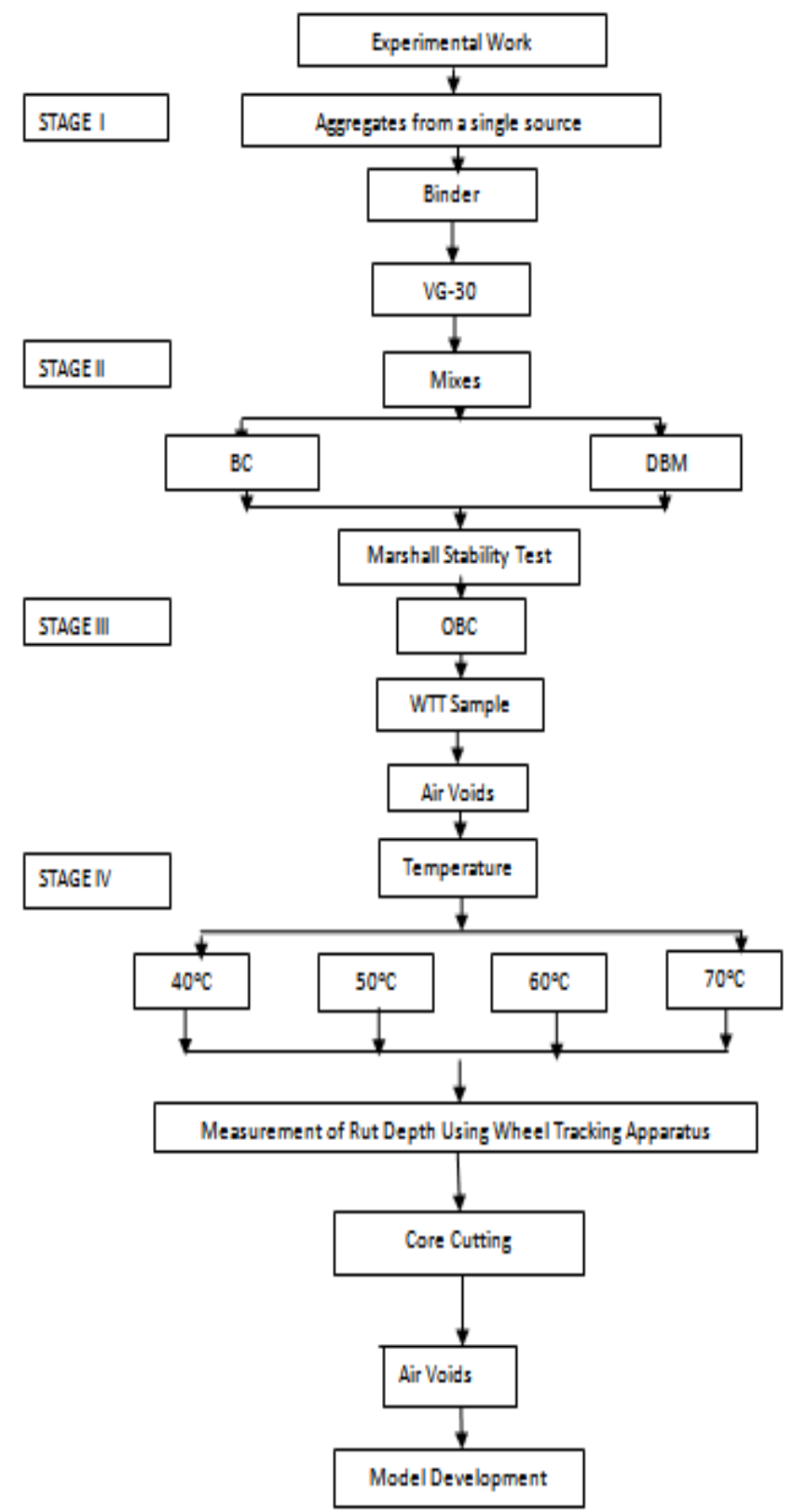

Fig 2 Flow Chat of Methodology

\section{LABORATORY INVESTIGATIONS AND DATA ANALYSIS GENERAL}

\subsection{General}

In this chapter details regarding laboratory investigations made for all the materials on isolation as well as a mix along with the analysis have been presented.

\subsection{Aggregates}

Aggregates are a component of asphalt concrete where in the aggregate acts like reinforcement to the overall composite material strength. The entire aggregate used in this study is from a single source. The aggregates physical properties are tabulated in Table 3.1. 
Table 3.2 Aggregate Physical properties

\begin{tabular}{|l|l|l|l|}
\hline $\begin{array}{l}\text { Sl. } \\
\text { No }\end{array}$ & Property & $\begin{array}{l}\text { Obtained } \\
\text { Value }\end{array}$ & $\begin{array}{l}\text { Specificat } \\
\text { ions }\end{array}$ \\
\hline 1 & $\begin{array}{l}\text { Combined Elongation } \\
\text { and Flakiness Index }\end{array}$ & 27 & $30 \mathrm{max}$ \\
\hline 2 & $\begin{array}{l}\text { Los Angeles Abrasion } \\
\text { Value }\end{array}$ & 28 & $30 \mathrm{max}$ \\
\hline 3 & Aggregate Impact Test & 20 & 30 max \\
\hline 4 & Specific Gravity Test & 2.58 & $2.5-3$ \\
\hline 5 & Water Absorption & 0.1 & 2 max \\
\hline 6 & Stripping Value & 95 & $95 \min$ \\
\hline
\end{tabular}

\subsection{Asphalt Binder}

The tests to assess the properties and requirements of paving grade asphalt are the viscosity tests, softening point test, ductility test and the penetration test. Also specific gravity test and flash and fire point tests are needed for use in paving applications. The properties of binders (VG-30) used in this experimentation are listed in Table 3.2.
Table 3.3 Conventional test results of asphalt binders

\begin{tabular}{|l|l|l|l|}
\hline $\begin{array}{l}\text { Sl. } \\
\text { No }\end{array}$ & Property & $\begin{array}{l}\text { Values } \\
\text { obtained }\end{array}$ & $\begin{array}{l}\text { Specific } \\
\text { ations }\end{array}$ \\
\cline { 3 - 4 } 1 & $\begin{array}{l}\text { PG-30 } \\
\left(\text { Penetration at } 25{ }^{\circ} \mathrm{C}, 0.1 \mathrm{~mm}\right.\end{array}$ & 67 & VG-30 \\
\hline 2 & $\begin{array}{l}\text { Softening Point }(\mathrm{R} \& \mathrm{~B}),{ }^{\circ} \mathrm{C} \\
(\text { SPR\&B) }\end{array}$ & 54 & $47, \mathrm{Min}$ \\
\hline 3 & Ductility (cm) & 50 & 70 \\
\hline 3 & $\begin{array}{l}\text { Elastic Recovery at } 15{ }^{\circ} \mathrm{C}, \\
\%\end{array}$ & 48 & 40, Min \\
\hline 4 & Flash Point (open cup), ${ }^{\circ} \mathrm{C}$ & 210 & 220 \\
\hline
\end{tabular}

\subsection{Marshall Stability Test}

The values obtained from Marshall Stability Test are discussed below

\subsubsection{Bituminous Concrete Mix using VG-30 binder}

Marshall Stability test was conducted on BC mix using VG30 binder and the results are displayed in the table 3.3.1 The Optimum Binder Content (OBC) is found to be $6 \%$.

\subsubsection{Dense Bituminous Macadam Mix using VG-30}

\section{Binder}

Marshall Stability test was conducted on DBM mix using VG-30 binder and the results are displayed in the table 3.3.2 The Optimum Binder Content (OBC) is found to be $5.75 \%$.

Table 3.4.1 Marshall Stability results for BC mix using VG-30

\begin{tabular}{|c|c|c|c|c|c|c|c|c|}
\hline \multirow[t]{2}{*}{$\begin{array}{l}\text { Bitumen } \\
(\%)\end{array}$} & \multicolumn{2}{|c|}{$\begin{array}{l}\text { Weight of the } \\
\text { Sample }\end{array}$} & \multirow{2}{*}{$\begin{array}{l}\text { Bulk Specific } \\
\text { Gravity }\left(G_{b}\right)\end{array}$} & \multirow{2}{*}{$\begin{array}{l}\% \text { Air Voids } \\
\left(V_{\mathrm{a}}\right)\end{array}$} & \multirow[t]{2}{*}{ VMA } & \multirow[t]{2}{*}{ VFB } & \multirow[t]{2}{*}{$\begin{array}{l}\text { Marshall Stability } \\
\text { (kg) }\end{array}$} & \multirow[t]{2}{*}{$\begin{array}{l}\text { Flow } \\
(\mathbf{m m})\end{array}$} \\
\hline & Air & Water & & & & & & \\
\hline 5 & 1188 & 692 & 2.25 & 5.32 & 18.62 & 85.47 & 2388 & 4.21 \\
\hline 5.5 & 1176 & 680 & 2.29 & 4.27 & 17.95 & 90.7 & 1891 & 3.24 \\
\hline 6 & 1188 & 670 & 2.27 & 2.5 & 18.25 & 79.63 & 1982 & 3.12 \\
\hline 6.5 & 1190 & 678 & 2.25 & 1.78 & 18.36 & 88.98 & 2184 & 4.51 \\
\hline 7 & 1184 & 686 & 2.3 & 2.16 & 17.22 & 82.86 & 2312 & 4.62 \\
\hline
\end{tabular}

Table 3.4.2 Marshall Stability results for DBM mix using VG-30

\begin{tabular}{|c|c|c|c|c|c|c|c|c|}
\hline \multirow{2}{*}{$\begin{array}{l}\text { Bitumen } \\
(\%)\end{array}$} & \multicolumn{2}{|c|}{$\begin{array}{l}\text { Weight of the } \\
\text { Sample }\end{array}$} & \multirow{2}{*}{$\begin{array}{l}\text { Bulk Specific } \\
\text { Gravity }\left(G_{b}\right)\end{array}$} & \multirow{2}{*}{$\begin{array}{l}\text { \% Air Voids } \\
\left(\mathbf{V}_{\mathbf{a}}\right)\end{array}$} & \multirow[t]{2}{*}{ VMA } & \multirow[t]{2}{*}{ VFB } & \multirow{2}{*}{$\begin{array}{l}\text { Marshall Stability } \\
\text { (kg) }\end{array}$} & \multirow{2}{*}{$\begin{array}{l}\text { Flow } \\
(\mathbf{m m})\end{array}$} \\
\hline & Air & Water & & & & & & \\
\hline 5 & 1186 & 658 & 2.25 & 6.06 & 17.29 & 64.95 & 1967 & 3.26 \\
\hline 5.5 & 1178 & 650 & 2.27 & 4.54 & 16.48 & 72.43 & 1815 & 2.93 \\
\hline 6 & 1180 & 656 & 2.25 & 5.13 & 17.52 & 70.70 & 2042 & 2.76 \\
\hline 6.5 & 1174 & 652 & 2.25 & 4.91 & 17.84 & 72.49 & 1891 & 3.51 \\
\hline 7 & 1190 & 666 & 2.27 & 3.63 & 17.26 & 78.95 & 1922 & 3.85 \\
\hline
\end{tabular}

\subsection{Wheel Tracking Test (WTT)}

Wheel tracking test developed by British National Rail Road Institute (BNRRI) used to study the rut characteristics of different HMA pavements and the rut depth characteristics of different sub grade materials. The wheel tracking test setup is as shown in Figure3.5. By this test can measure the rut depth and number of passes to failure. Slabs have been prepared with several combinations to determine the effect of binder type (VG-30), effect of binder content (OBC) used to prepare the mix, mix type $(\mathrm{BC}, \mathrm{DBM})$ and effect of temperature $\left(40,50,60\right.$, and $\left.70{ }^{\circ} \mathrm{C}\right)$. The test matrix of the slabs taken with various combinations is given in Table 3.5 


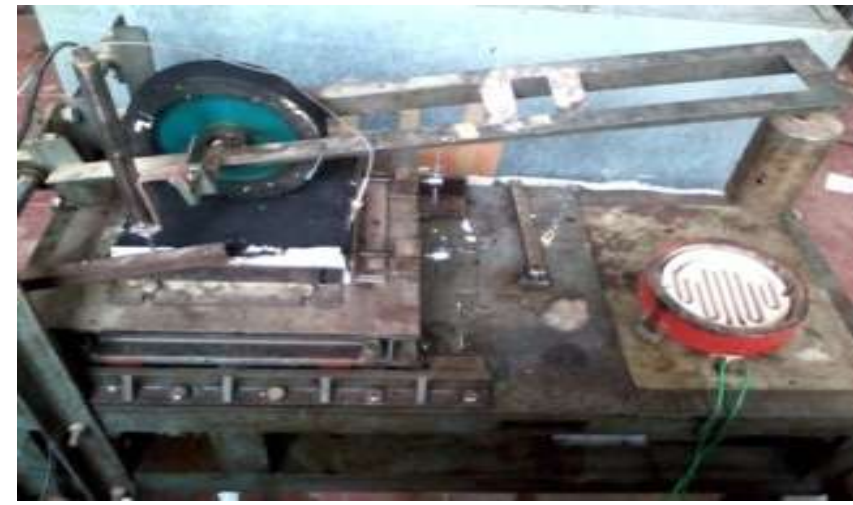

Fig 3.5 Wheel tracking testing machine

Table 3.5 Test matrix considered in experimental work

\begin{tabular}{|l|l|l|l|l|l|l|}
\hline $\begin{array}{l}\text { Sa } \\
\text { mp } \\
\text { le } \\
\text { No }\end{array}$ & mix & $\begin{array}{l}\text { Gr } \\
\text { ad } \\
\text { ing }\end{array}$ & $\begin{array}{l}\text { Binder } \\
\text { Type }\end{array}$ & $\begin{array}{l}\text { OB } \\
\mathbf{( \% )}\end{array}$ & $\begin{array}{l}\text { Tempe } \\
\text { rature }\end{array}$ & $\begin{array}{l}\text { Compa } \\
\text { ction } \\
\text { Durati } \\
\text { on(min } \\
\text { ) }\end{array}$ \\
\hline 1 & BC & 2 & VG-30 & 6 & 40 & 5 \\
\hline 2 & BC & 2 & VG-30 & 6 & 50 & 5 \\
\hline 3 & BC & 2 & VG-30 & 6 & 60 & 5 \\
\hline 4 & BC & 2 & VG-30 & 6 & 70 & 5 \\
\hline 5 & BC & 2 & VG-30 & 6 & 40 & 10 \\
\hline 6 & BC & 2 & VG-30 & 6 & 50 & 10 \\
\hline 7 & BC & 2 & VG-30 & 6 & 60 & 10 \\
\hline 8 & BC & 2 & VG-30 & 6 & 70 & 10 \\
\hline 9 & BC & 2 & VG-30 & 6 & 40 & 15 \\
\hline 10 & BC & 2 & VG-30 & 6 & 50 & 15 \\
\hline 11 & BC & 2 & VG-30 & 6 & 60 & 15 \\
\hline 12 & DBM & 2 & VG-30 & 6 & 70 & 15 \\
\hline 13 & DBM & 2 & VG-30 & 5.75 & 40 & 5 \\
\hline 14 & DBM & 2 & VG-30 & 5.75 & 50 & 5 \\
\hline 15 & DBM & 2 & VG-30 & 5.75 & 60 & 5 \\
\hline 16 & DBM & 2 & VG-30 & 5.75 & 70 & 5 \\
\hline 17 & DBM & 2 & VG-30 & 5.75 & 40 & 10 \\
\hline 18 & DBM & 2 & VG-30 & 5.75 & 50 & 10 \\
\hline 19 & DBM & 2 & VG-30 & 5.75 & 60 & 10 \\
\hline 20 & DBM & 2 & VG-30 & 5.75 & 70 & 10 \\
\hline 21 & DBM & 2 & VG-30 & 5.75 & 40 & 15 \\
\hline 22 & DBM & 2 & VG-30 & 5.75 & 50 & 15 \\
\hline 23 & DBM & 2 & VG-30 & 5.75 & 60 & 15 \\
\hline 24 & DBM & 2 & VG-30 & 5.75 & 70 & 15 \\
\hline & & & & & & \\
\hline
\end{tabular}

After the WTT, cores were extracted from the samples to know the air voids. Four cores had been cut from the slabs. Using the OBC obtained from the Marshall Stability test wheel tracking samples were casted and tested. The permanent deformation occurred after 30,000 repetitions $\left(\mathrm{RD}_{30,000}\right)$ was measured. $\mathrm{RD}_{30,000}$ is used to compare the performance of the $\mathrm{BC}$ and $\mathrm{DBM}$ mixes tested at different temperatures $\left(40,50,60\right.$, and $\left.70{ }^{\circ} \mathrm{C}\right)$ and with binder type VG-30. Using the rutting performance parameter $\left(\mathrm{RD}_{30,000}\right)$, the effect of temperature and air voids are investigated.

A sample table to know the permanent deformation values after every 200 passes for BC mix prepared using VG-30 and tested at $60{ }^{\circ} \mathrm{C}$ is shown in Table 3.6
Table 3.6 Rut depths as a function of Number of passes for BC prepared using VG-30 binder

\begin{tabular}{|c|c|c|c|}
\hline $\begin{array}{ll}\text { No. } & \text { of } \\
\text { Passes } & \\
\end{array}$ & $\begin{array}{l}\text { Rut } \\
\text { Depth }\end{array}$ & No. of Passes & Rut Depth \\
\hline 0 & 0 & 15800 & 3.3193 \\
\hline 200 & 0.2127 & 14600 & 3.2415 \\
\hline 400 & 0.2976 & 14800 & 3.2571 \\
\hline 600 & 0.3401 & 15000 & 3.2843 \\
\hline 800 & 0.3826 & 15200 & 3.2999 \\
\hline 1000 & 0.4252 & 15400 & 3.3038 \\
\hline 1200 & 0.5102 & 15600 & 3.3154 \\
\hline 1400 & 0.6378 & 15800 & 3.3193 \\
\hline 1600 & 0.7654 & 16000 & 3.3232 \\
\hline 1800 & 0.8929 & 16200 & 3.3232 \\
\hline 2000 & 1.0205 & 16400 & 3.3349 \\
\hline 2200 & 1.0630 & 16600 & 3.3427 \\
\hline 2400 & 1.1481 & 16800 & 3.3505 \\
\hline 2600 & 1.1906 & 17000 & 3.3544 \\
\hline 2800 & 1.2331 & 17200 & 3.3582 \\
\hline 3000 & 1.2757 & 17400 & 3.3621 \\
\hline 3200 & 1.3607 & 17600 & 3.3699 \\
\hline 3400 & 1.4456 & 17800 & 3.3971 \\
\hline 3600 & 1.4882 & 18000 & 3.4010 \\
\hline 3800 & 1.5732 & 18200 & 3.4166 \\
\hline 4000 & 1.6583 & 18400 & 3.4283 \\
\hline 4200 & 1.7008 & 18600 & 3.4361 \\
\hline 4400 & 1.7434 & 18800 & 3.4477 \\
\hline 4600 & 1.7859 & 19000 & 3.4516 \\
\hline 4800 & 1.8284 & 19200 & 3.4672 \\
\hline 5000 & 1.9135 & 19400 & 3.4711 \\
\hline 5200 & 1.9984 & 19600 & 3.4788 \\
\hline 5400 & 2.0835 & 19800 & 3.4827 \\
\hline 5600 & 2.1260 & 20000 & 3.4983 \\
\hline 5800 & 2.2111 & 20200 & 3.5022 \\
\hline 6000 & 2.3387 & 20400 & 3.5139 \\
\hline 6200 & 2.3502 & 20600 & 3.5255 \\
\hline 6400 & 2.3812 & 20800 & 3.5333 \\
\hline 6600 & 2.4237 & 21000 & 3.5411 \\
\hline 6800 & 2.5088 & 21200 & 3.5528 \\
\hline 7000 & 2.6362 & 21400 & 3.5683 \\
\hline 7200 & 2.6788 & 21600 & 3.5761 \\
\hline 7400 & 2.6974 & 21800 & 3.5839 \\
\hline 7600 & 2.7194 & 22000 & 3.5956 \\
\hline 7800 & 2.7213 & 22200 & 3.6033 \\
\hline 8000 & 2.7229 & 22400 & 3.6189 \\
\hline 8200 & 2.7421 & 22600 & 3.6267 \\
\hline 8400 & 2.7638 & 22800 & 3.6345 \\
\hline 8600 & 2.7734 & 23000 & 3.6461 \\
\hline 8800 & 2.7953 & 23200 & 3.6539 \\
\hline 9000 & 2.8063 & 23400 & 3.6695 \\
\hline 9200 & 2.8184 & 23600 & 3.6734 \\
\hline 9400 & 2.8283 & 23800 & 3.6850 \\
\hline 9600 & 2.8489 & 24000 & 3.7084 \\
\hline 9800 & 2.8588 & 24200 & 3.7123 \\
\hline 10000 & 2.8832 & 24400 & 3.7278 \\
\hline 10200 & 2.8917 & 24600 & 3.7356 \\
\hline 10400 & 2.9059 & 24800 & 3.7434 \\
\hline 10600 & 2.9339 & 25000 & 3.7590 \\
\hline
\end{tabular}




\begin{tabular}{|l|l|l|l|}
10800 & 2.9503 & 25200 & 3.7667 \\
\hline 11000 & 2.9583 & 25400 & 3.7784 \\
\hline 11200 & 2.9765 & 25600 & 3.7823 \\
\hline 11400 & 2.9831 & 25800 & 3.7979 \\
\hline 11600 & 2.9913 & 26000 & 3.8173 \\
\hline 11800 & 3.0106 & 26200 & 3.8212 \\
\hline 12000 & 3.0190 & 26400 & 3.8329 \\
\hline 12200 & 3.0309 & 26600 & 3.8484 \\
\hline 12400 & 3.0371 & 26800 & 3.8523 \\
\hline 12600 & 3.0615 & 27000 & 3.8679 \\
\hline 12800 & 3.0805 & 27200 & 3.8718 \\
\hline 13000 & 3.0917 & 27400 & 3.8834 \\
\hline 13200 & 3.1015 & 27600 & 3.8951 \\
\hline 13400 & 3.1170 & 27800 & 3.9029 \\
\hline 13600 & 3.1598 & 28000 & 3.9146 \\
\hline 13800 & 3.1715 & 28200 & 3.9224 \\
\hline 14000 & 3.1948 & 28400 & 3.9340 \\
\hline 14200 & 3.2182 & 28600 & 3.9418 \\
\hline 14400 & 3.2260 & 28800 & 3.9590 \\
\hline 14600 & 3.2415 & 29000 & 3.9629 \\
\hline 14800 & 3.2571 & 29200 & 3.9707 \\
\hline 15000 & 3.2843 & 29400 & 3.9846 \\
\hline 15200 & 3.2999 & 29600 & 4.0076 \\
\hline 15400 & 3.3038 & 29800 & 4.0158 \\
\hline 15600 & 3.3154 & 30000 & 4.0254 \\
\hline
\end{tabular}

Similar procedure is adopted for all the other 23 specimens and graphs are plotted for rut depth against number of observe to know the three permanent deformation response.

\subsubsection{Bituminous Concrete Mix using VG-30 Binder}

\section{Tested at $40^{\circ} \mathrm{C}$}

Figure 3.5.1 shows the graph plotted between the rut depth in $\mathrm{mm}$ and the number of repetitions for BC mix prepared with $6 \%$ VG-30 binder and compacted for 5 minutes. WTT was carried out at $40{ }^{\circ} \mathrm{C}$ for this mix. This sample refers to $1^{\text {st }}, 9^{\text {th }}$ sample in the test matrix. The rut depth after 30,000 repetitions $\left(R_{30,000}\right)$ is $5.48569 \mathrm{~mm}$.

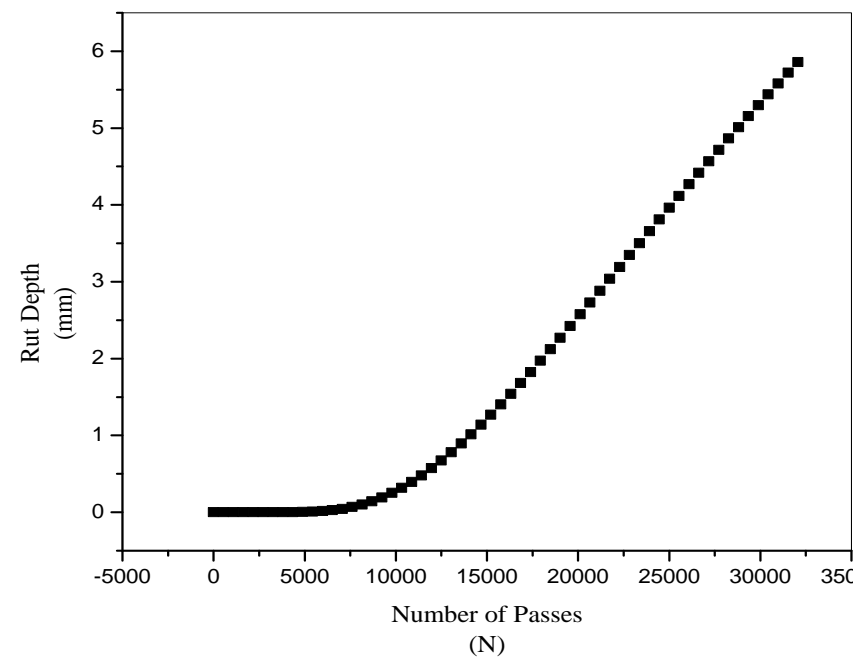

Fig 3.5.1 Rut depth as a function of number of passes for BC using VG-30 binder at $40^{\circ} \mathrm{C}$

\subsubsection{Dense Bituminous Macadam mix using VG-30} tested at $40^{\circ} \mathrm{C}$

Figure 3.5.2 shows the graph plotted between the rut depth in $\mathrm{mm}$ and the number of repetitions for DBM mix prepared with $5.75 \%$ VG-30 binder and 5 minutes compaction. WTT is carried out at $40{ }^{\circ} \mathrm{C}$ for this mix. This sample refers to thirteenth sample in the test matrix given in Table 3.7. The rut depth after 30,000 repetitions $\left(\mathrm{RD}_{30,000}\right)$ is $5.99762 \mathrm{~mm}$.

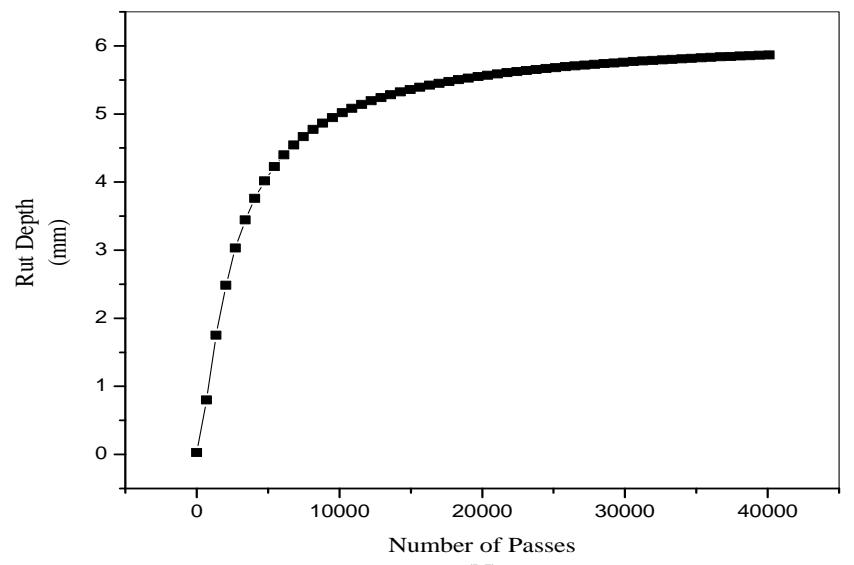

Fig. 3.5.2 Rut depth as a function of number of passes for $\mathrm{BC}$ using VG-30 binder at $40{ }^{\circ} \mathrm{C}$

\subsubsection{Effect of Temperature on rutting} characteristics of BC mix using VG-30

Figure 3.5.3 shows effect of permanent deformation characteristics of $\mathrm{BC}$ mix prepared using VG-30 at 5 minutes compaction.

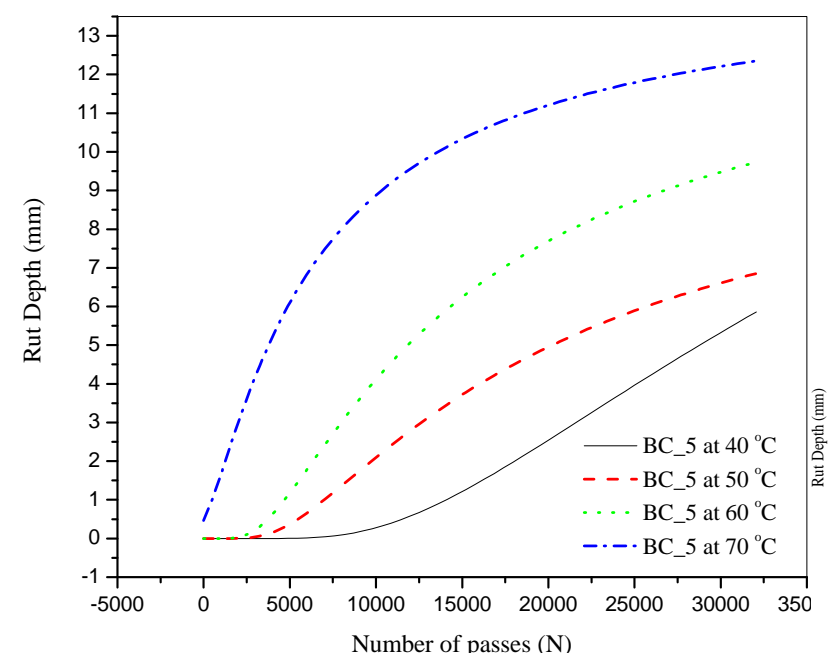

Fig 3.5.3 Rut depth plots showing effect of temperature for BC-VG-30

\subsubsection{Effect of Temperature on rutting characteristics of DBM mix using VG-30:}

Figure 3.5.4 shows effect of permanent deformation characteristics of DBM mix prepared using VG-30 at 5 \& 15 minutes compaction. 


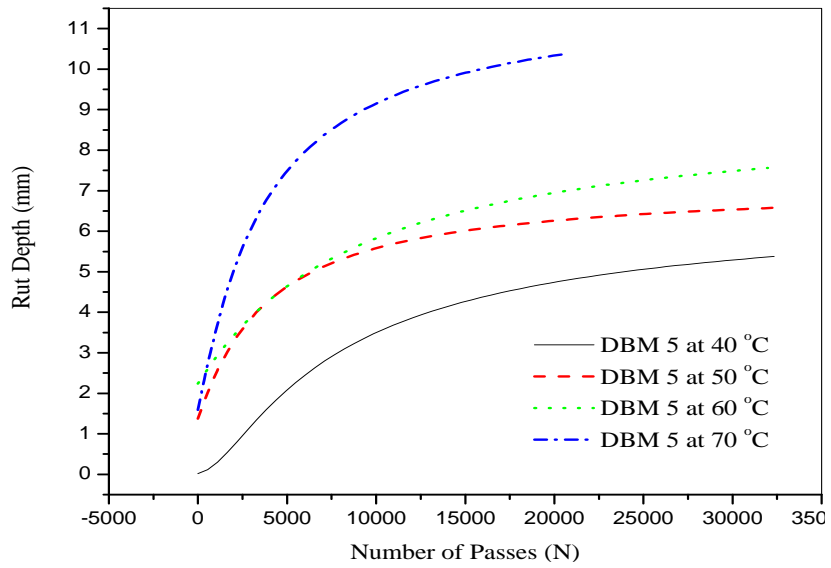

Fig 3.5.4 Rut depth plots showing effect of temperature for DBM-VG-30

\subsubsection{Core Cutting}

Core cutting is done to know the air voids of the sample. The results obtained from the core cutting samples are tabulated as below in Table 3.5.5

Table 3.5.5 Core cutting results

\begin{tabular}{|l|l|l|}
\hline Sample & $\begin{array}{l}\text { Compaction } \\
\text { Duration (min) }\end{array}$ & Air voids \\
\hline BC-VG-30 & 5 & 5.5475 \\
\hline BC-VG-30 & 10 & 4.0825 \\
\hline BC-VG-30 & 15 & 2.2775 \\
\hline DBM-VG-30 & 5 & 4.7925 \\
\hline DBM -VG-30 & 10 & 3.5075 \\
\hline DBM -VG-30 & 15 & 2.7775 \\
\hline
\end{tabular}

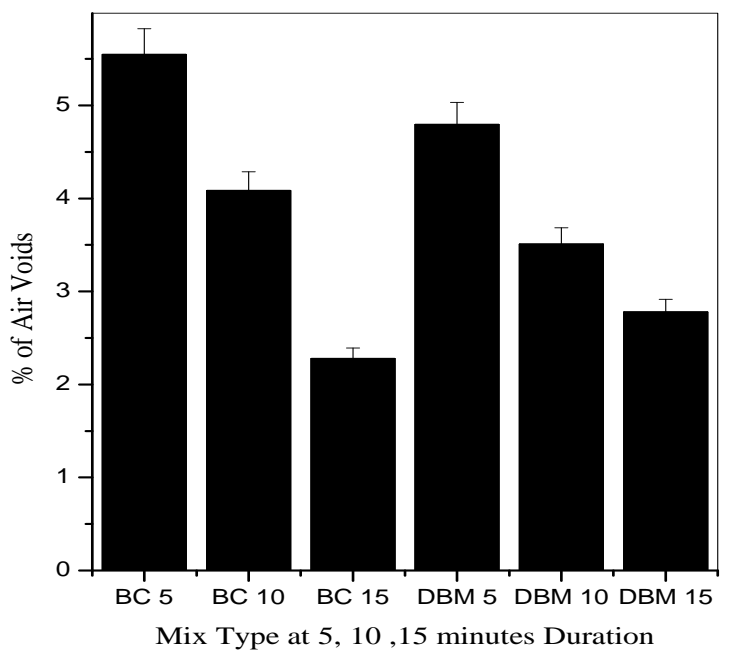

Fig 3.5.5 Comparison of air voids BC and DBM at different Compaction levels

\subsubsection{Summary}

In this chapter, the results obtained through laboratory investigations on asphalt mixes by varying mix type, test temperature, and air voids are discussed. Results obtained through laboratory investigations on asphalt binders and aggregates are also presented. Table 3.5.6 gives the brief details of the results of the dissertation work.

Table 3.5.6 Detailed results of the work

\begin{tabular}{|l|l|l|l|l|l|l|l|}
\hline S.NO & Mix & Grading & Binder Type & OBC $(\boldsymbol{\%})$ & Temperature & Rut Depth (mm) & Compaction Duration(min) \\
\hline 1 & BC & 2 & VG-30 & 6 & 40 & 5.48569 & 5 \\
\hline 2 & BC & 2 & VG-30 & 6 & 50 & 6.08976 & 5 \\
\hline 3 & BC & 2 & VG-30 & 6 & 60 & 7.6248 & 5 \\
\hline 4 & BC & 2 & VG-30 & 6 & 70 & 10.67828 & 5 \\
\hline 5 & BC & 2 & VG-30 & 6 & 40 & 2.89964 & 10 \\
\hline 6 & BC & 2 & VG-30 & 6 & 50 & 3.499982 & 10 \\
\hline 7 & BC & 2 & VG-30 & 6 & 60 & 7.09998 & 10 \\
\hline 8 & BC & 2 & VG-30 & 6 & 70 & 7.48962 & 10 \\
\hline 9 & BC & 2 & VG-30 & 6 & 40 & 0.89942 & 15 \\
\hline 10 & BC & 2 & VG-30 & 6 & 50 & 2.69982 & 15 \\
\hline 11 & BC & 2 & VG-30 & 6 & 60 & 4.49968 & 15 \\
\hline 12 & BC & 2 & VG-30 & 6 & 70 & 5.89764 & 15 \\
\hline 13 & DBM & 2 & VG-30 & 5.75 & 40 & 5.59962 & 5 \\
\hline 14 & DBM & 2 & VG-30 & 5.75 & 50 & 6.96452 & 5 \\
\hline 15 & DBM & 2 & VG-30 & 5.75 & 60 & 9.68822 & 5 \\
\hline 16 & DBM & 2 & VG-30 & 5.75 & 70 & 12.09632 & 5 \\
\hline 17 & DBM & 2 & VG-30 & 5.75 & 40 & 3.89041 & 10 \\
\hline 18 & DBM & 2 & VG-30 & 5.75 & 50 & 4.72956 & 10 \\
\hline 19 & DBM & 2 & VG-30 & 5.75 & 60 & 6.00912 & 10 \\
\hline 20 & DBM & 2 & VG-30 & 5.75 & 70 & 8.89996 & 10 \\
\hline 21 & DBM & 2 & VG-30 & 5.75 & 40 & 4.35642 & 15 \\
\hline 22 & DBM & 2 & VG-30 & 5.75 & 50 & 4.86249 & 15 \\
\hline 23 & DBM & 2 & VG-30 & 5.75 & 60 & 5.12628 & 15 \\
\hline 24 & DBM & 2 & VG-30 & 5.75 & 70 & 5.49982 & 15 \\
\hline & & & & & & & \\
\hline
\end{tabular}




\section{DEVELOPMENT OF RUT DEPTH MODEL}

\subsection{General}

In this chapter, we have discussed the simplest way to develop a three-stage permanent deformation model and presented.

\subsection{Three Stage Equations}

As discussed earlier in the literature review the three stage equations are shown in equations (4.1) to (4.4).

Primary Stage

$$
\varepsilon_{p}=a N^{b}, N<N_{P S}
$$

Secondary Stage

$$
\varepsilon_{p}=\varepsilon_{P S}+c\left(N-N_{P S}\right), N_{\mathrm{PS}} \leq N<N_{S T}
$$

Tertiary Stage

$$
\begin{aligned}
& \boldsymbol{\varepsilon}_{p}=\boldsymbol{\varepsilon}_{S T}+d\left(e^{f\left(N-N_{S T}\right)}-1\right), N \geq N_{S T} \\
& \boldsymbol{\varepsilon}_{S T}=\varepsilon_{P S}+c\left(N_{S T}-N_{P S}\right)
\end{aligned}
$$

Where,

$\varepsilon_{p}=$ permanent strain,

$N=$ number load repetitions

$\mathrm{a}, \mathrm{b}, \mathrm{c}, \mathrm{d}$ and e are = material constant,

$N_{P S}=$ number of load repetitions related to the initiation of secondary stage,

$\varepsilon_{P S}=$ permanent strain related to the initiation of secondary stage,

$N_{S T}=$ number of load repetitions related to the initiation of tertiary stage and

$\varepsilon_{S T}=$ permanent strain related to the initiation of tertiary stage.

Applying these equations to the data presented in the

\begin{tabular}{|c|c|c|c|}
\hline Sample & Primary Stage Equation & $\begin{array}{l}\text { Secon } \\
\text { dary } \\
\text { Stage } \\
\text { Equa } \\
\text { tion }\end{array}$ & $\begin{array}{l}\text { Tertiar } \\
\text { y Stage } \\
\text { Equatio } \\
\text { n }\end{array}$ \\
\hline $\begin{array}{l}40^{\circ} \mathrm{C} \text { at } \\
5 \mathrm{~min}\end{array}$ & $\begin{array}{l}\mathcal{E}_{p}=0.004 * N^{0.699} \\
, \mathrm{R}^{2}=0.914\end{array}$ & None & None \\
\hline $\begin{array}{l}50^{\circ} \mathrm{C} \text { at } \\
5 \mathrm{~min}\end{array}$ & $\begin{array}{l}\varepsilon_{p}=0.568 * N^{0.241} \\
, \mathrm{R}^{2}=0.940\end{array}$ & None & None \\
\hline $\begin{array}{l}60^{\circ} \mathrm{C} \text { at } \\
5 \mathrm{~min}\end{array}$ & $\begin{array}{l}\mathcal{E}_{p}=0.412 * N^{0.284} \\
, \mathrm{R}^{2}=0.989\end{array}$ & None & None \\
\hline $\begin{array}{l}70^{\circ} \mathrm{C} \text { at } \\
5 \mathrm{~min}\end{array}$ & $\begin{array}{l}\mathcal{E}_{p}=0.413 * N^{0.332} \\
, \mathrm{R}^{2}=0.957\end{array}$ & None & None \\
\hline $\begin{array}{l}40^{\circ} \mathrm{C} \text { at } \\
10 \mathrm{~min}\end{array}$ & $\begin{array}{l}\mathcal{E}_{p}=0.001 * N^{0.766} \\
, \mathrm{R}^{2}=0.996\end{array}$ & None & None \\
\hline $\begin{array}{l}50^{\circ} \mathrm{C} \text { at } \\
10 \mathrm{~min}\end{array}$ & $\begin{array}{l}\boldsymbol{\varepsilon}_{p}=0.067 * N^{0.379} \\
, \mathrm{R}^{2}=0.911\end{array}$ & None & None \\
\hline $\begin{array}{l}60^{\circ} \mathrm{C} \text { at } \\
10 \mathrm{~min}\end{array}$ & $\begin{array}{l}\mathcal{E}_{p}=0.015 * N^{0.591} \\
\mathrm{R}^{2}=0.992\end{array}$ & None & None \\
\hline $\begin{array}{l}70^{\circ} \mathrm{C} \text { at } \\
10 \mathrm{~min}\end{array}$ & $\begin{array}{l}\boldsymbol{E}_{p}=0.178 * N^{0.385} \\
, \mathrm{R}^{2}=0.993\end{array}$ & None & None \\
\hline $\begin{array}{l}40^{\circ} \mathrm{C} \text { at } \\
15 \mathrm{~min}\end{array}$ & $\begin{array}{l}\mathcal{E}_{p}=0.029 * N^{0.334} \\
, \mathrm{R}^{2}=0.885\end{array}$ & None & None \\
\hline $\begin{array}{l}50^{\circ} \mathrm{C} \text { at } \\
15 \mathrm{~min}\end{array}$ & $\begin{array}{l}\mathcal{E}_{p}=0.506 * N^{0.168} \\
, \mathrm{R}^{2}=0.881\end{array}$ & None & None \\
\hline $\begin{array}{l}60^{\circ} \mathrm{C} \text { at } \\
15 \mathrm{~min}\end{array}$ & $\begin{array}{l}\mathcal{E}_{p}=0.114 * N^{0.354} \\
, \mathrm{R}^{2}=0.846\end{array}$ & None & None \\
\hline $\begin{array}{l}70^{\circ} \mathrm{C} \text { at } \\
15 \mathrm{~min}\end{array}$ & $\begin{array}{l}\boldsymbol{E}_{p}=0.448 * N^{0.251} \\
\mathrm{R}^{2}=0.832\end{array}$ & None & None \\
\hline
\end{tabular}
previous chapter resulted in the rut depth equations as shown in as Table 4.1. \& Table 4.2
Table 4.1 Three stage equations for BC-VG-30

Table 4.2 Three stage equations for DBM-VG-30

\begin{tabular}{|l|l|l|l|}
\hline Sample & Primary Stage Equation & $\begin{array}{l}\text { Secon } \\
\text { dary } \\
\text { Stage } \\
\text { Equa } \\
\text { tion }\end{array}$ & $\begin{array}{l}\text { Tertiar } \\
\text { y Stage } \\
\text { Equatio } \\
\mathbf{n}\end{array}$ \\
\hline $\begin{array}{l}40^{\circ} \mathrm{C} \\
5 \mathrm{~min}\end{array}$ & $\begin{array}{l}\boldsymbol{E}_{p}=0.006^{*} N^{0.724} \\
, \mathrm{R}^{2}=0.934\end{array}$ & None & None \\
\hline $\begin{array}{l}50^{\circ} \mathrm{C} \text { at } \\
5 \mathrm{~min}\end{array}$ & $\begin{array}{l}\boldsymbol{E}_{p}=0.325^{*} N^{0.172} \\
, \mathrm{R}^{2}=0.993\end{array}$ & None & None \\
\hline
\end{tabular}




\begin{tabular}{|c|c|c|c|}
\hline $\begin{array}{l}60^{\circ} \mathrm{C} \text { at } \\
5 \mathrm{~min}\end{array}$ & $\begin{array}{l}\mathcal{E}_{p}=0.018^{*} N^{0.372} \\
, \mathrm{R}^{2}=0.922\end{array}$ & None & None \\
\hline $\begin{array}{l}70^{\circ} \mathrm{C} \text { at } \\
5 \mathrm{~min}\end{array}$ & $\begin{array}{l}\mathcal{E}_{p}=0.082 * N^{0.495} \\
, \mathrm{R}^{2}=0.937\end{array}$ & None & None \\
\hline $\begin{array}{l}40^{\circ} \mathrm{C} \text { at } \\
10 \mathrm{~min}\end{array}$ & $\begin{array}{l}\mathcal{E}_{p}=0.003^{*} N^{0.633} \\
, \mathrm{R}^{2}=0.996\end{array}$ & None & None \\
\hline $\begin{array}{l}50^{\circ} \mathrm{C} \text { at } \\
10 \mathrm{~min}\end{array}$ & $\begin{array}{l}\boldsymbol{\varepsilon}_{p}=0.04 * N^{0.445} \\
\mathrm{R}^{2}=0.996\end{array}$ & None & None \\
\hline $\begin{array}{l}60^{\circ} \mathrm{C} \text { at } \\
10 \mathrm{~min}\end{array}$ & $\begin{array}{l}\mathcal{E}_{p}=0.358^{*} N^{0.271} \\
, \mathrm{R}^{2}=0.778\end{array}$ & None & None \\
\hline $\begin{array}{l}70^{\circ} \mathrm{C} \text { at } \\
10 \mathrm{~min}\end{array}$ & $\begin{array}{l}\boldsymbol{\varepsilon}_{p}=0.07 * N^{0.453} \\
, \mathrm{R}^{2}=0.992\end{array}$ & None & None \\
\hline $\begin{array}{l}40^{\circ} \mathrm{C} \text { at } \\
15 \mathrm{~min}\end{array}$ & $\begin{array}{l}\boldsymbol{\varepsilon}_{p}=0.087 * N^{0.382} \\
, \mathrm{R}^{2}=0.821\end{array}$ & None & None \\
\hline $\begin{array}{l}50^{\circ} \mathrm{C} \text { at } \\
15 \mathrm{~min}\end{array}$ & $\begin{array}{l}\mathcal{E}_{p}=0.113 * N^{0.372} \\
, \mathrm{R}^{2}=0.799\end{array}$ & None & None \\
\hline $\begin{array}{l}60^{\circ} \mathrm{C} \text { at } \\
15 \mathrm{~min}\end{array}$ & $\begin{array}{l}\boldsymbol{E}_{p}=0.142 * N^{0.356} \\
, \mathrm{R}^{2}=0.778\end{array}$ & None & None \\
\hline $\begin{array}{l}70^{\circ} \mathrm{C} \text { at } \\
15 \mathrm{~min}\end{array}$ & $\begin{array}{l}\varepsilon_{p}=0.190 * N^{0.330} \\
, \mathrm{R}^{2}=0.778\end{array}$ & None & None \\
\hline
\end{tabular}

\subsection{Model Development}

By the laboratory experimentation on asphalt mixtures, we can get the relationship between the number of load applications and permanent deformation in three distinct stages, namely the primary, secondary and tertiary stages. From the laboratory studies, the data collected is used as the database for the model development. Multiple regression analysis is used for model development. Rut depth being the dependent parameter, air voids and temperature are the independent parameters as shown in Equation 4.5. Table 4.3 shows properties of asphalt mixes and asphalt. Rut depth also depends on the type of binder. In this analysis only one binder (VG-30) is used.

$$
\text { Rut Depth }=\mathrm{a}_{0}+\mathrm{a}_{1} *(\text { temperature })+\mathrm{a}_{2} *(\text { air voids })
$$

Where, $\mathrm{a}_{0}=$ intercept, $\mathrm{a}_{1}$ and $\mathrm{a}_{2}=$ variables corresponding temperature and air voids respectively.

The results of multiple regression analysis are tabulated in the Table 4.4
Table 4.3 Parameters for carrying out multiple regression analysis

\begin{tabular}{|c|c|c|c|c|c|c|c|}
\hline $\begin{array}{l}\text { Sa } \\
\text { m } \\
\text { pl } \\
\text { e } \\
\text { No }\end{array}$ & Mix & $\begin{array}{l}\text { Gr } \\
\text { ad } \\
\text { ing }\end{array}$ & $\begin{array}{l}\text { Binder } \\
\text { Type }\end{array}$ & $\begin{array}{l}\text { OB } \\
C \\
(\%)\end{array}$ & $\begin{array}{l}\text { Te } \\
\mathbf{m} \\
\mathbf{p}\end{array}$ & $\begin{array}{l}\text { Rut } \\
\text { Depth } \\
(\mathbf{m m})\end{array}$ & $\begin{array}{l}\text { Com } \\
\text { p } \\
\text { actio } \\
\text { n } \\
\text { (min) }\end{array}$ \\
\hline 1 & $\mathrm{BC}$ & 2 & VG-30 & 6 & 40 & 5.48569 & 5 \\
\hline 2 & $\mathrm{BC}$ & 2 & VG-30 & 6 & 50 & 6.08976 & 5 \\
\hline 3 & $\mathrm{BC}$ & 2 & VG-30 & 6 & 60 & 7.6248 & 5 \\
\hline 4 & $\mathrm{BC}$ & 2 & VG-30 & 6 & 70 & 10.67828 & 5 \\
\hline 5 & $\mathrm{BC}$ & 2 & VG-30 & 6 & 40 & 2.89964 & 10 \\
\hline 6 & $\mathrm{BC}$ & 2 & VG-30 & 6 & 50 & 3.499982 & 10 \\
\hline 7 & $\mathrm{BC}$ & 2 & VG-30 & 6 & 60 & 7.09998 & 10 \\
\hline 8 & $\mathrm{BC}$ & 2 & VG-30 & 6 & 70 & 7.48962 & 10 \\
\hline 9 & $\mathrm{BC}$ & 2 & VG-30 & 6 & 40 & 0.89942 & 15 \\
\hline 10 & $\mathrm{BC}$ & 2 & VG-30 & 6 & 50 & 2.69982 & 15 \\
\hline 11 & $\mathrm{BC}$ & 2 & VG-30 & 6 & 60 & 4.49968 & 15 \\
\hline 12 & $\mathrm{BC}$ & 2 & VG-30 & 6 & 70 & 5.89764 & 15 \\
\hline 13 & DBM & 2 & VG-30 & 5.75 & 40 & 5.59962 & 5 \\
\hline 14 & DBM & 2 & VG-30 & 5.75 & 50 & 6.96452 & 5 \\
\hline 15 & DBM & 2 & VG-30 & 5.75 & 60 & 9.68822 & 5 \\
\hline 16 & DBM & 2 & VG-30 & 5.75 & 70 & 12.09632 & 5 \\
\hline 17 & DBM & 2 & VG-30 & 5.75 & 40 & 3.89041 & 10 \\
\hline 18 & DBM & 2 & VG-30 & 5.75 & 50 & 4.72956 & 10 \\
\hline 19 & DBM & 2 & VG-30 & 5.75 & 60 & 6.00912 & 10 \\
\hline 20 & DBM & 2 & VG-30 & 5.75 & 70 & 8.89996 & 10 \\
\hline 21 & DBM & 2 & VG-30 & 5.75 & 40 & 4.35642 & 15 \\
\hline 22 & DBM & 2 & VG-30 & 5.75 & 50 & 4.86249 & 15 \\
\hline 23 & DBM & 2 & VG-30 & 5.75 & 60 & 5.12628 & 15 \\
\hline 24 & DBM & 2 & VG-30 & 5.75 & 70 & 5.49982 & 15 \\
\hline
\end{tabular}

Table 4.4 multiple regression analysis results

\begin{tabular}{|c|c|c|c|c|}
\hline Mix & $\begin{array}{l}\text { Independent } \\
\text { Variable }\end{array}$ & $\begin{array}{l}\text { Regression } \\
\text { Parameter } \\
\text { S }\end{array}$ & $\begin{array}{l}\text { Parameter } \\
\text { Values }\end{array}$ & $\mathbf{R}^{2}$ \\
\hline \multirow{3}{*}{$\mathrm{BC}$} & Intercept & $a_{0}$ & -43.7252 & \multirow{3}{*}{$\begin{array}{l}0.98 \\
8512\end{array}$} \\
\hline & Temperature & $\mathrm{a}_{1}$ & 0.196356 & \\
\hline & Air Voids & $a_{2}$ & 7.46221 & \\
\hline \multirow{3}{*}{ DBM } & Intercept & $\mathrm{a}_{0}$ & -8.98442 & \multirow{3}{*}{$\begin{array}{l}0.97 \\
5161\end{array}$} \\
\hline & Temperature & $a_{1}$ & 0.219971 & \\
\hline & Air Voids & $a_{2}$ & 1.221589 & \\
\hline
\end{tabular}

Multiple regression analysis of BC and DBM resulted in Equations 4.6 and 4.7 respectively.

Rut depth $=0.196356($ Temperature $)+7.46221$ (Air Voids $)-$ 43.7252

Rut depth $=0.219971($ Temperature $)+1.221589$ (Air Voids) $-8.98442$

\section{SUMMARY AND CONCLUSION}

\subsection{General}

In this chapter summary and conclusions of the study are presented. The scope for the future research work is also discussed. 


\subsection{Summary}

In this study an attempt has been made to develop rut depth model prediction with respect to temperature, air voids and binder type. Literature review on the fundamentals of rutting and the influence of binder type, temperature, zero shear, air voids are presented. Review of earlier studies revealed that binder type, air voids, temperature have significant influence on rutting. Rutting behaviour of BC and DBM mix prepared with VG-30 binder and compacted for 5, 10 and 15 minutes and tested at temperatures $40,50,60$, and $70{ }^{\circ} \mathrm{C}$ is reported in this in this work. Plots were drawn for permanent deformation against number wheel passes and comparisons are made considering the effect of temperature and air voids. Finally a model is formulated with rut depth as a function of temperature and air voids using multiple linear regression analysis.

\section{CONCLUSION}

Following are the conclusions drawn from the present study:

i. Temperature had a significant effect on rutting potential. Rutting resistance decreases with increase in temperature.

ii. Air voids also had a significant effect on rutting potential. Rutting increases with decrease in compaction duration.

iii. Good correlation was observed between rutting potential, temperature and air voids. Multiple regression analysis gave an $\mathrm{R}^{2}$ value of 0.98 for $\mathrm{BC}$ and 0.97 for DBM.

\section{SCOPE FOR FUTURE RESEARCH WORK}

The scope for further research work is summarized below:

i. The present study can be repeated with several binder types of unmodified, PMB, CRMB, and NRMB because harder binders are more viscous and more resistant to rutting. Aged binders can be used to determine the effect of aging.

ii. The present study can be repeated with different mix types such as BC, SDBC, SMA, DBM which are currently being used in India. All existing gradation types can be used to prepare the samples. The performance of asphalt mixes can be studied by adding fillers, and/or fibres (coconut or jute).

iii. Mixes can be prepared by using optimum binder content (OBC), less than OBC and more than OBC to compute the three stage permanent deformation response.

\section{REFERENCES}

[1] Archilla, A. R., (2006) "Use of Superpave Gyratory Compaction Data for Rutting Prediction" Journal of Transportation Engineering, Vol. 132, 734-741.

[2] Adria'n Ricardo Archilla1 and SamerMadanat (2000) "Development Of A Pavement Rutting Model From Experimental Data" Journal of Transportation Engineering, Vol. 126, 291-299.

[3] Adriana Vargas-Nordcbeck\& David H. Timm (2012) "Rutting characterization of warm mix asphalt and high RAP mixtures" Road Materials and Pavement Design Vol. 13, 1-20
[4] Ahmed Ebrahim, Abu El-MaatyBehiry (2012) "Fatigue and rutting lives in flexible pavement" Highways and Airports Engineering 2012.

[5] Ahmad, J., Rahman, M. Y., and Hainin, M. R. (2011). "Rutting evaluation of dense graded hot mix asphalt mixture." International Journal of Engineering \& Technology, 11(05), 56-60.

[6] Amir Golalipoura, EhsanJamshidib, YunusNiazic, Zahra Afsarykiad, MahmoodKhadem (2012) "Effect of Aggregate Gradation on Rutting of Asphalt Pavements" Social and Behavioral Sciences 53; 440 - 449.

[7] Asi, I. M. (2006). "Laboratory comparison study for the use of stone matrix asphalt in hot weather climates." Construction and Building Materials, 20, 982-989. 